\title{
Corticotropin-Releasing Hormone Signaling in the Oval Bed Nucleus of the Stria Terminalis Mediates Chronic Stress-Induced Negative Valence Behaviors Associated with Anxiety
}

Pu Hu a, Isabella Maita a, Christopher Kwok a, Edward Gu a, Mark Gergues a, Ji Liu b, Zhiping Pang b, Dick F. Swaab c Paul J. Lucassen d, Troy A. Roepke e, Benjamin A. Samuels a*

a: Department of Psychology, Rutgers University, Piscataway, NJ 08854, USA

b: Department of Neuroscience and Cell Biology, Rutgers Robert Wood Johnson Medical School, New Brunswick, NJ 08901, USA

c: Department of Neuropsychiatric Disorders, Netherlands Institute for Neuroscience, An Institute of the Royal Netherlands Academy of Arts and Sciences, Meibergdreef, Amsterdam, 1105BA, The Netherlands

d: Brain Plasticity Group, Swammerdam Institute for Life Sciences, Center for Neuroscience, University of Amsterdam, Sciencepark 904, 1098 XH Amsterdam, The Netherlands

e: Department of Animal Sciences, School of Environmental and Biological Sciences, Rutgers University, New Brunswick, NJ 08901, USA

\section{*: Corresponding author: Dr. Benjamin Samuels (ben.samuels@rutgers.edu)}

Abbreviated Title: CRH Signaling in ovBNST Mediates Chronic Stress Effects 


\begin{abstract}
:
The bed nucleus of stria terminalis (BNST) is a forebrain region highly sensitive to stress that expresses corticotropin-releasing hormone ( $\mathrm{CRH}$ ) neuropeptide at high levels and regulates negative valence behaviors associated with anxiety. However, how chronic stress modulates $\mathrm{CRH}$ signaling and neuronal activity in BNST remains unknown. We subjected C57BL6/J mice to a 6-week chronic variable mild stress (CVMS) paradigm and investigated the effects on behavior, BNST cellular neurophysiology, and BNST CRH signaling. We also utilized pharmacological infusions and optogenetics to decipher and mimic the effects of CVMS on BNST cellular neurophysiology and behavior. CVMS elevated plasma corticosterone levels, induced negative valence behaviors associated with anxiety, diminished $\mathrm{M}$-currents (voltage-gated $\mathrm{K}^{+}$currents that stabilize membrane potential and regulate neuronal excitability), and increased mEPSC amplitude in ovBNST. Additionally, the number of $\mathrm{c}^{-} \mathrm{fos}^{+}, \mathrm{CRH}^{+}$, and $\mathrm{CRH}$ activator pituitary adenylate cyclase-activating polypeptide $(\mathrm{PACAP})^{+}$cells were increased while $\mathrm{CRH}$ inhibitor striatal-enriched protein tyrosine phosphatase $(\text { STEP })^{+}$cells were decreased in ovBNST. These expression data were confirmed with qPCR. CVMS also activated PKA in BNST and the electrophysiological and behavioral effects of CVMS were reversed by ovBNST infusion of the PKA-selective antagonist H89. Moreover, optogenetic activation of ovBNST directly induced negative valence behaviors associated with anxiety, mimicking the effects of CVMS. CVMS mediates effects on negative valence behaviors associated with anxiety by activating CRH signaling components and cellular excitability in ovBNST. Our findings decipher an important $\mathrm{CRH}$-associated stress molecular signature in BNST and advance our understanding of the neural circuitry underlying stress-induced disorders.
\end{abstract}




\section{Introduction:}

Stressful environments promote vigilance, which, when moderate, is adaptive and essential for survival. However, chronic exposure to stressful experiences or environments can be maladaptive and increase risk assessment of low imminence threats, which is a feature of persistent anxiety. The limbic forebrain structure bed nucleus of the stria terminalis (BNST) is critical for mediating the neuroendocrine stress response (1) and potential threat regulation (2-5). Overall, BNST integrates stress and reward information from the limbic system and projects to neuroendocrine and autonomic neural systems located in the hypothalamus and brain stem regions that mediate the hypothalamic-pituitary-adrenal (HPA) stress response $(3,5,6)$. Stimulating BNST elicits anxiogenic responses (7), whereas BNST inactivation is anxiolytic $(8,9)$. Consequently, BNST dysfunction contributes to exaggerated stress responses and stress-related mood disorders.

The stress hormone corticotropin-releasing hormone (CRH or CRF) is abundantly expressed in hypothalamic paraventricular nucleus (PVN) parvocellular neurons. These PVN CRH neurons regulate HPA axis activity (10) and cortisol release (11-13). Importantly, $\mathrm{CRH}$ is also highly expressed in BNST $(5,14-18)$, especially in the oval nucleus (19). CRH mRNA in the antero-dorsolateral BNST (BNSTadl) is increased by stress exposure (20).

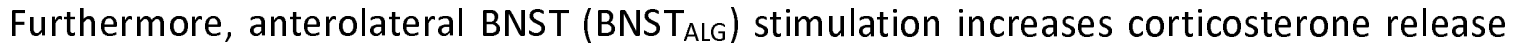
$(6,21)$. Notably, BNST orchestrates stress responses in a $\mathrm{CRH}$-dependent fashion (22). CRH neurons in BNSTadl mediate anxiogenic effects $(19,23,24)$ and negative affective responses to stress (25-28). CRH injections into BNST and CRH overexpression in BNST cause anxiogenic effects (26) and result in increased negative valence behaviors associated with anxiety $(29,30)$. Therefore, CRH dysfunction in BNST likely contributes to stress-related behavioral states (31) and mood disorders (32).

BNST is a complex conglomerate structure with a heterogeneous population of neurons $(19,33-35)$. Within $\mathrm{BNST}_{\mathrm{ALG}}$, the highest concentration of $\mathrm{CRH}$ neurons is 
located in the oval nucleus $(14,19,36)$ (ovBNST). ovBNST is thought to be a master controller of BNST outflow that regulates overall BNST activity (3). Optogenetic inhibition of ovBNST deceases, whereas stimulation promotes negative valence behaviors (37). Here, we utilize a chronic variable mild stress (CVMS; also known as chronic unpredictable mild stress) paradigm in mice to better understand the effects of chronic stress on BNST. CVMS and other chronic stress paradigms are widely used to determine the neuroendocrine and physiological effects that result in behavioral disturbances in stress-related mood disorders (38) (39). However, little is known about how chronic stress modulates the expression of $\mathrm{CRH}$ signaling components and cellular neurophysiology properties in the BNST and whether these alterations underlie chronic stress effects on behavior. Here we demonstrate that CVMS modulates expression of $\mathrm{CRH}$-related stress signaling components (including its upstream activator, pituitary adenylate cyclase-activating polypeptide (PACAP) as well as its inhibitor, striatal-enriched protein tyrosine phosphatase (STEP)) and neuronal activity as measured by both M-currents (a voltage-dependent non-inactivating $\mathrm{K}^{+}$current that stabilizes cellular membrane potential) and miniature excitatory postsynaptic currents (mEPSCs) in mouse OvBNST. Furthermore, we show that selective inhibition of PKA la downstream modulator of $\mathrm{CRH}$ signaling) in ovBNST reverses CVMS effects on M-currents, mEPSCS, and behavior. Furthermore, optogenetic activation of ovBNST mimics CVMS effects on behavior. Taken together, these data suggest that the effects of CVMS on negative valence behaviors associated with anxiety are mediated through altered $\mathrm{CRH}$ signaling and neuronal activity in ovBNST. 


\section{METHODS AND MATERIALS:}

Mice:

All procedures were in accordance with institutional guidelines based on the National Institutes of Health standards and approved by the Rutgers Institutional Animal Care and Use Committee. All mice used (except for optogenetics) were adult male C57BL/6J mice purchased from Jackson. For optogenetics, dopamine receptor D1a (Drd1a)-Cre (GENSAT line EY266) transgenic mice were generated from in house breeding.

Except for CVMS exposure, all mice were maintained in a controlled temperature $\left(22^{\circ} \mathrm{C}\right)$ and a $12 \mathrm{~h} \mathrm{light/dark}$ cycle with food and water provided ad libitum.

\section{Chronic variable mild stress (CVMS):}

CVMS was performed as described $(40,41)$. CVMS began when mice were 6 weeks old and persisted for 6 weeks. 50 mice were randomly assigned to non-stress (Control) $(n=25)$ or CVMS groups $(n=25)$. Variable mild stressors were used: daily bedding alterations (repeated sawdust changes, removal of sawdust, damp sawdust, substitution of sawdust with $21{ }^{\circ} \mathrm{C}$ water), cage-tilting ( $45^{\circ}$ angle), predator sounds (15 min), cage shift (placed into the empty cage of another male), alterations of the light/dark cycle, lights off for $180 \mathrm{~min}$, overnight food/water deprivation $(40,41)$.

50 total mice were used in 3 sets of experiments. 20 were used for behavior $(n=10$ per group) and subsequent immunohistochemistry (IHC) (randomly selected $n=6$ per group), 12 for electrophysiology ( $n=6$ per group), and 18 for plasma CORT measurements ( $n=7-9$ per group) and real-time quantitative PCR analysis ( $q-P C R)$ ( $n=7-9$ per group).

\section{Data Analysis:}

All data are presented as mean \pm SEM. Statistical analysis were conducted with GraphPad Prism (La Jolla, CA). Comparisons of M-current I-V plots between Control and 
Stress group were performed at each voltage $(-25$ to $-75 \mathrm{mV})$ using a one-way ANOVA with posthoc Newman-Keuls comparisons. Maximum current at $-35 \mathrm{mV}$ was analyzed with paired Student's $t$-test. For mEPSCs, amplitude and frequency were analyzed off-line using Mini Analysis (Synaptosoft, Fort Lee, NJ). mEPSC amplitude and frequency comparisons were performed using paired Student's $t$-test. For behavior, IHC, and plasma CORT concentration comparisons between Control and CVMS groups, data were analyzed with a one-way ANOVA and posthoc Tukey comparisons. For optogenetics behavior experiments, data were analyzed using a two-way ANOVA with posthoc Tukey comparisons, with light and group as independent factors.

Differences were considered significant when $p<0.05 . n$ represents the number of cells or animals.

Additional methods and materials are provided in the Supplemental File. 


\section{RESULTS}

We first exposed a cohort of male C57BL6/J mice to either chronic variable mild stress (CVMS) or control conditions. CVMS began when mice were 6 weeks old and the paradigm persisted for 6 weeks (Fig.1A). 6 weeks of CVMS resulted in a significant percent decrease in body weight compared with non-stress controls $(F(1,13)=84.908$, $\mathrm{p}<0.001)$. We next sampled blood plasma and found significantly higher basal CORT levels in the CVMS group relative to the control group $(F(1,18)=12.281, p<0.01)$ (Fig.S1).

We next assessed behaviors that are influenced by chronic stress exposure. Mice exposed to CVMS showed decreased open arm duration in elevated plus maze (EPM) $(F(1,18)=8.262 ; p=0.01 ; F i g .1 B)$, decreased sucrose preference in sucrose preference test (SPT) $(F(1,18)=23.118 ; p<0.001 ; F i g .1 D)$, decreased center distance $(F(1,18)=30.155$; $p<0.001$; Fig.1E), center duration $(F(1,18)=59.24 ; p<0.001$; Fig.1F), and center entries $(F(1,18)=19.50 ; p=0.001 ; F i g .1 G)$ in open field (OF), and increased latency to eat in novelty suppressed feeding (NSF) $(F(1,18)=10.460 ; p<0.01$; Fig.1H) relative to non-stress controls. EPM open arm entries $(F(1,18)=2.065 ; p=0.17$; Fig.1C) and forced swim test (FST) immobility $(F(1,18)=3.035 ; p=0.08$; Fig.1I) were not significantly affected by CVMS. Taken together, these data demonstrate that our CVMS protocol effectively induces negative valence behaviors associated with anxiety in EPM, OF, and NSF, and decreases reward valuation in the anhedonia-related SPT.

Next, we determined the effects of CVMS on ovBNST electrophysiological properties using ex vivo slices (Fig.2A). We first measured M-currents (KCNQ/Kv7 channels), a subthreshold noninactivating voltage-dependent outward $\mathrm{K}^{+}$current that controls action potential generation and neuronal excitability (42), in ovBNST neurons using a standard deactivation-activation protocol over a voltage range ( -75 to $-25 \mathrm{mV}$ ) where M-currents have profound effects on neuronal excitability (Fig.2C). M-currents were calculated by determining current relaxation, the difference between the instantaneous and steady states (Fig.3C arrows). The maximum M-currents were 
recorded at $-35 \mathrm{mV}$ (Fig.2B). 20 min of recording did not show a M-current rundown (amplitude decrease) (example traces in Fig.2B; $n=5$ ). Application of the KCNQ/Kv7 channel blocker XE991 robustly decreased M-currents (Fig.2B). We next determined the role of M-currents in modulating BNST neuronal excitability. To this end, firing activity of ovBNST neurons was continuously monitored in current clamp mode (Fig.2D). XE991 application induced action potentials after $30 \mathrm{~s}$, and 6-7 min of XE991 perfusion led to robust firing bursts indicative of activation and hyperexcitability (Fig.2D).

Interestingly, the outward M-current was attenuated in ovBNST neurons from CVMS mice (Fig.2E), especially at higher voltages ( $p=0.005,0.003,0.001,0.004,0.003$, and 0.005 at $-50,-45,-40,-35,-30$ and $-25 \mathrm{mV}$, respectively; $n=6)$, with a significant effect of stress $(F(1,10)=16.353, p=0.002)$. At $-35 \mathrm{mV}$, the outward $M$-current peak value was robustly decreased from $202.59 \pm 39.95 \mathrm{pA}$ in control to $42.90 \pm 16.54 \mathrm{pA}(\mathrm{t}=3.693$; $\mathrm{p}=0.004)$ in CVMS slices. Furthermore, CVMS significantly depolarized the resting membrane potential (RMP) ( $t=3.192, p=0.023$ ) (Fig.2F) and increased the input resistance (Rin) ( $t=3.632, p=0.014)$ (Fig.2G) in ovBNST neurons.

Reduced M-currents can elicit increased excitatory cellular responses to synaptic inputs (43). Therefore, we hypothesized that CVMS may alter excitatory glutamatergic neurotransmission in ovBNST. Indeed, CVMS significantly increased the average amplitude of mEPSCs (Fig.2H-J) $(t=3.141, p=0.012)$ without affecting mEPSC frequency $(t=0.790, p=0.451$ (Fig.2J). Taken together, these data demonstrate that CVMS contributes to increased excitability and hyperactivation of ovBNST neurons.

Corticotropin-releasing hormone $(\mathrm{CRH})$, a neuropeptide that is released from the paraventricular nucleus (PVN) of the hypothalamus (PVN) in response to stress, is also highly expressed in BNST (Fig.3.1A). Therefore, we next assessed CVMS effects on CRH signaling in BNST. To this end, we subdivided anterior-dorsolateral BNST (BNSTadI) into the oval nucleus of BNST (OvBNST) and the surrounding anterolateral dorsal region of BNST (adBNST) (Fig.S4E). CVMS increased the number of $\mathrm{CRH}^{+}$cells in BNSTadl and 
ovBNST relative to control (BNSTadl: $F(1,10)=49.78, p<0.01$; ovBNST: $F(1,10)=28.93$; $\mathrm{p}<0.01$ ) (Fig.3.2C-D). However, the number of $\mathrm{CRH}^{+}$cells in adBNST did not differ between CVMS and control (Fig.S4.2A).

We next assessed c-fos in the BNST of control and CVMS mice as a marker of neuronal activation (c-fost cell example in Fig.3.1B). The number of c-fos-immunoreactive cells in BNSTadl and ovBNST were significantly increased by CVMS (BNSTadl: $\quad F(1,10)=24.896, \quad p<0.01 ; \quad$ ovBNST: $F(1,10)=65.29, p<0.001$ ) (Fig.3.2A-B). However, $\mathrm{c}^{-\mathrm{fos}^{+}}$cells were unchanged by CVMS in the adBNST region (Fig.S4.2B).

Consistent with these data, the $\mathrm{CRH}$ activator pituitary adenylate cyclase-activating polypeptide (PACAP) (Fig.3.1C), was more highly expressed in BNSTadl and ovBNST after CVMS, while the $\mathrm{CRH}$ inhibitor striatal-enriched protein tyrosine phosphatase (STEP) (Fig.3.1D), was decreased in BNSTadl and ovBNST of CVMS mice (PACAP BNSTadl: $F(1,10)=77.89, p<0.01$; PACAP ovBNST: $F(1,10)=98.36, p<0.001$ (shown in Fig.3.2.E and F)); STEP BNSTadl: $F(1,8)=16.063, p<0.01$; STEP ovBNST: $F(1,8)=20.099, p<0.01$ ) (Fig.3.2G-H). However, the number of PACAP ${ }^{+}$and STEP ${ }^{+}$cells in adBNST was unchanged by CVMS (Fig.S4C-D). Taken together, these data demonstrate that $\mathrm{CRH}$ signaling is increased in BNSTadl and ovBNST by CVMS.

To complement these immunohistochemistry results, we next assessed mRNA expression of $\mathrm{CRH}$, PACAP, STEP, and the CRH receptors CRHR1 and CRHR2 in BNSTadl by qPCR (Fig.4A). CVMS significantly increased CRH $(F(1,14)=6.303, p=0.018$; Fig.4B) and PACAP $(F(1,12)=5.597, p=0.037 ; F i g .4 C)$ and decreased STEP $(F(1,16)=5.877, p=0.031$; Fig.4D) expression in BNSTadl relative to non-stress controls. Interestingly, CRHR1 expression was also significantly increased in CVMS mice $(F(1,15)=4.985, p=0.041$; Fig.4E), whereas CRHR2 was unchanged $(F(1,14)=0.750, p=0.403$; Fig.4F). CVMS did not affect KCNQ2, KCNQ3, and KCNQ5 subunit (the Kv7 M-channel components) expression (Fig.S2). Since these qPCR results suggest that CRHR1 expression is selectively increased in the BNST by CVMS, and CRHR1 is a Gs protein-coupled receptor linked to adenylyl 
cyclase (AC), we next assessed whether CVMS resulted in protein kinase $A$ (PKA) activation in BNST. To this end, we assessed protein expression levels of PKA (using an antibody recognizing the PKA subunit $C-\alpha$ ) and its activated form p-PKA (using an antibody recognizing Thr197 phosphorylated-PKA-C; Fig.4G) in BNSTadl tissue punches from CVMS and control mice by western blot. While total PKA levels did not differ $(t=1.46, p=0.171)$ (Fig.4I), p-PKA expression in BNSTadl was increased by CVMS ( $t$ test $\mathrm{t}=5.105, \mathrm{p}=0.0003$ ) (Fig.4J), indicating CVMS increases PKA activation in BNSTadl.

We also assessed STEP protein expression in BNSTadl tissue punches (Fig.4H). Whereas the membrane isoform STEP61 did not differ ( $t=1.060, p=0.31$ ) (Fig.4L), CVMS decreased expression of the cytosolic isoform STEP46 in BNSTadl was decreased in CVMS mice $(t=2.292, p=0.026)$ (Fig.4K), suggesting CVMS specifically affects cytosolic STEP46 expression in BNST.

We next assessed whether BNST PKA activation mediates the effects of CVMS on M-currents and mEPSCs. When ex vivo brain slices were pre-incubated with the selective PKA antagonist $\mathrm{H} 89$ for $30 \mathrm{~min}$, the CVMS-induced decrease in ovBNST outward M-currents was significantly attenuated at higher voltages $(F(1,10)=16.353, p=0.002$; Fig.5A) ( $p=0.044,0.045,0.047,0.042$ and 0.020 at $-45,-40,-35,-30$ and $-25 \mathrm{mV}$, respectively). At $-40 \mathrm{mV}$, the outward $M$-current peak value was increased $(t=2.292$, $\mathrm{p}=0.045$ ) in CVMS slices pre-incubated with H89 compared with CVMS slices (Fig.5A). Similarly, the CVMS-induced mEPSC amplitude increase in ovBNST was significantly decreased $(t=2.913, p=0.025)$ when slices were preincubated with the selective PKA antagonist $\mathrm{H} 89$ for 30 min (Fig.5B). By contrast, mEPSC frequency in ovBNST was not different between CVMS and CVMS+H89 slices (t=0.210; $p=0.843$ ) (Fig.5C). Importantly, H89 pre-incubation had no significant effects on ovBNST M-currents and mEPSCs in control slices (Fig.S8). Taken together, these results suggest that the effects of CMVS on M-currents and mEPSC amplitude in ovBNST are mediated by PKA activation.

Next, we examined whether PKA activation also mediates the behavioral effects of 
CVMS by infusing the PKA-selective antagonist H89 (25 nM, dissolved in $0.5 \mu$ l saline) into ovBNST (Fig.6A-B). BNST H89 infusions into CVMS mice significantly increased EPM open arm duration $(F(1,14)=12.528 ; \quad p=0.011 ; F i g .6 C)$, OF center distance $(F(1,15)=27.962 ; p<0.01 ; F i g .6 E)$, OF center duration $(F(1,15)=31.752 ; p<0.01 ; F i g .6 F)$, OF center entries $(\mathrm{F}(1,15)=18.121 ; \mathrm{p}<0.01 ; \mathrm{Fig} .6 \mathrm{G})$, and SPT sucrose preference $(F(1,19)=4.43 ; p=0.035 ; F i g .6 H)$, and decreased NSF latency to eat $(F(1,19)=4.434$; $\mathrm{p}=0.025$ ) (Fig.6I) relative to control infusions into CVMS mice. H89 did not affect EPM open arm entries in CVMS mice $(F(1,14)=1.212 ; p=0.374 ;$ Fig.6D). Importantly, H89 infusions into BNST had no effects in EPM, SPT, OF, or NSF in control mice (Fig.S9). Taken together, these results demonstrate that CVMS effects on negative valence behaviors associated with anxiety in tasks such as EPM, OF, and NSF and reward valuation in the anhedonia-related SPT are mediated by ovBNST PKA activation.

Since our data demonstrate that CVMS decreases M-currents and increases mEPSC amplitude in BNST, we next sought to determine whether selective activation of ovBNST mimics the behavioral effects of CVMS. To this end, we injected either a Cre-inducible ChR2 (AAV5-EF1 $\alpha$-DIO-ChR2(H134R)-eYFP) or control virus (AAV5-EF1 $\alpha$-DIO-eYFP) into BNST of dopamine receptor D1a (Drd1a)-Cre transgenic mice (Fig.7A). Within BNST, Cre expression in Drd1a-Cre mice is restricted to ovBNST (37). Fig.7B-C shows expression of Control-eYFP (B) and ChR2-eYFP virus (C) in ovBNST. Similar to a previous report (Kim et al 2013), ChR2 photoillumination in ovBNST resulted in a significant virus $x$ light interaction for EPM open arm duration and entries (Fig.7D-E), (Duration: $F(2,42)=3.277$, $p=0.048$; Entries: $F(2,48)=8.746, p=0.001$ ). Specifically, ovBNST activation (light ON period) decreased open arm duration and entries relative to the preceding (Duration: p<0.01; Fig.7D; Entries: $p<0.01$; Fig.7E) and succeeding (Duration: $p=0.018$; Entries: $p<0.01$ ) light OFF periods. By contrast, there were no significant changes in open arm duration or entries across the light OFF/ON/OFF periods in mice injected with control virus. Similarly, in OF, ChR2 photoillumination in ovBNST resulted in a significant virus $x$ 
light interaction for center distance (Fig.7F), center duration (Fig.7G), and center entries (Fig.7H) (Distance: $F(2,39)=6.853, p=0.003$; Duration: $F(2,39)=4.72, p=0.015$; Entries: $F(2,36)=11.495, p<0.001)$. Specifically, ovBNST activation decreased these OF center measures relative to the preceding ( $p<0.01$ for all center measures) and succeeding ( $p<0.01$ for all center measures) light OFF periods (Fig.7F-H). By contrast, there were no changes in center measures across the light OFF/ON/OFF periods in mice injected with control virus. Taken together, these data demonstrate that selective activation of ovBNST neurons mimics the behavioral effects of CVMS and that ovBNST activation is sufficient to induce negative valence behaviors. 


\section{DISCUSSION}

Chronic stress results in long-term alterations in HPA axis activity and negative valence behaviors associated with anxiety (44-47). However, the neurobiological underpinnings remain elusive. BNST plays an important role in mediating sustained negative valence behaviors in rodents $(8,48,49)$ and mood disorders in humans $(50-54)$, and BNST dysfunction is implicated in stress-related psychopathology (2-4). Importantly, our data demonstrate that CVMS alters expression of $\mathrm{CRH}$-related stress signaling components (including PACAP, STEP, and CRHR1) and enhances cellular excitability (manifested by decreased M-current and increased mEPSC amplitude) in ovBNST neurons. These CVMS-induced alterations result in BNST dysfunction, which in turn contributes to negative valence behavioral disturbances associated with anxiety. Direct optogenetic activation of ovBNST neurons mimics behavioral effects of CVMS, further suggesting that BNST dysfunction may underlie CVMS-mediated behavioral disturbances.

The CVMS paradigm is well validated to elicit negative valence behaviors. Whereas chronic exposure to homotypic stressors usually engenders HPA habituation (55-58), this shortcoming is circumvented by the CVMS paradigm, in which rodents were exposed to randomly alternating stressors in an unpredictable manner (59). In this study, a robust behavioral phenotype and elevated plasma corticosterone levels were found after a 6-week CVMS exposure. It will be informative for future studies to determine whether other chronic stress paradigms, such as chronic social defeat stress, elicit similar effects on OVBNST.

\section{M-current and mEPSC regulation by CVMS}

The $\mathrm{M}$-current is a subthreshold voltage-dependent non-inactivating outward $\mathrm{K}^{+}$ current that stabilizes membrane potential and sets cellular threshold for action potential firing $(60,61)$. M-channels are composed of Kv7 subunits (KCNQ) (62), open at 
resting membrane potential, and undergo conformational changes that facilitate even more opening during depolarization, which results in membrane potential clamping. Therefore, M-currents function as a brake on repetitive action potential charges (62) and play an essential role in controlling neuronal excitability (63). Diminished M-currents allow neurons to fire more rapidly (61), as evidenced during XE991 (M-channel selective blocker) perfusion. Our results show that CVMS-induced decreased M-currents in ovBNST are accompanied by a more depolarized cellular resting membrane potential (Fig.2F), indicating enhanced neuronal excitability. Acute restraint suppresses $\mathrm{M}$-currents and increases cellular activity in $\mathrm{CRH}$ neurons in the hypothalamic PVN, which may contribute to HPA hyperactivation after acute stress (64). However, whether chronic stress modulates $M$-currents was previously unknown. Our study provides a direct cellular mechanism by which CVMS increases excitability of ovBNST neurons. Consequently, targeting ovBNST M-channels may provide a potential therapeutic strategy to treat stress-related mood disorders (65).

Our qPCR results revealed no significant changes in KCNQ subunit mRNA expression after CVMS (Fig.S2), so post-translational modifications such as phosphorylation may account for M-current inhibition. We found that CVMS effects on M-currents are mediated in part through post-translational PKA activation (Fig.4J). Although few studies have assessed direct regulation of KCNQ channel activity by PKA, M-currents can be inhibited by phosphorylation (15). Anchoring proteins, such as members of the A-kinase anchoring protein family (AKAP), may bind to and localize activated PKA into a membrane signaling complex near the KCNQ channel subunit in order to modulate channel function (17) (66). Similarly, increased amplitude (but not frequency) of mEPSCS after CVMS suggests a postsynaptic effect, such as PKA-mediated phosphorylation of the GluR1 subunit of AMPA receptors $(24,67)$. GluR1 phosphorylation is closely correlated with increased receptor surface trafficking and membrane redistribution $(68,69)$, and increased AMPA receptor density on postsynaptic membranes would increase mEPSC 
amplitude (reflecting increased synaptic responses) (70-72).

\section{CVMS-induced alterations in BNST CRH signaling}

Although hypothalamic $\mathrm{CRH}$ signaling was not assessed here, previous studies show PVN CRH neuron activation (73) and increased PVN CRH expression in rats exposed to CVMS (74). In BNST, CRH signaling is linked to negative affective states $(25,26,75-78)$. Increased CRH concentrations $(79,80)$ and mRNA levels $(20,81)$ are found in rat BNST after chronic stress, however whether chronic stress modulates $\mathrm{CRH}$ neuropeptides in mouse BNST was unknown. Notably, the oval nuclei in the $\mathrm{BNST}_{\mathrm{ALG}}$, which resides dorsal to the anterior commissure (19), harbors the highest concentration of $\mathrm{CRH}$ neurons in BNST $(14,36)$. Local CRH signaling in ovBNST is suggested to be stress responsive $(28,35$, 82). Importantly, we also compared CRH expression in the anterodorsal BNST region (adBNST) surrounding ovBNST. Interestingly, CVMS selectively increases the number of CRH-immunopositive cells in ovBNST (with no changes in adBNST). In addition, CVMS selectively increases CRHR1 mRNA expression in BNSTadl, suggesting the effects of CRH are mediated mainly through this receptor. This is consistent with recent studies demonstrating stress effects on behavior depend on CRHR1 receptor activation (22) and are blocked by CRHR1 antagonist injections into BNST $(25,26)$. CRHR1 and CRHR2 subtypes play opposing roles during the stress response $(44,47)$, as CRHR1 activation initiates the stress response and results in negative valence behaviors $(25,26,83-85)$, whereas CRHR2 activation in the BNST mainly functions to terminate the stress response and facilitate stress recovery $(86,87)$. Thus, exaggerated CRHR1 expression and a disruption in the expression balance of these two receptors may dampen stress-coping capability and precipitate stress-related psychopathology.

We also assessed the effects of CVMS on pituitary adenylate cyclase-activating polypeptide (PACAP) and striatal-enriched protein tyrosine phosphatase (STEP). PACAP is a key stress regulator (88-90) that is upstream of $\mathrm{CRH}$ and stimulates $\mathrm{CRH}$ production 
and secretion (91). PACAP dysregulation is implicated in depression (92) and PTSD in humans (93). PACAP is highly expressed in BNST and PACAP-containing neurons in ovBNST closely interact with $\mathrm{CRH}$-containing neurons as part of the stress response. Chronic stress increases PACAP expression in the dorsal BNST $_{A L G}(81,94)$, and PACAP infusion into BNST increases plasma corticosterone concentrations (95). Treatment with a PAC1 receptor antagonist attenuates behavioral and endocrine effects of stress (94). We found that increased CRH and CRHR1 levels were accompanied by increased PACAP expression in ovBNST following CVMS. We also investigated the effects of CVMS on STEP (also known as protein tyrosine phosphatase nonreceptor type 5, PTPN5). STEP is a brain-specific tyrosine phosphatase that dephosphorylates and inactivates several kinases (including ERK1/2 and p38MAPK, Fyn) (96) and STEP overexpression enhances stress resilience (97). Furthermore, STEP loss-of-function increases susceptibility to stress-induced cognitive deficits (98). Downregulation of STEP expression in BNST $\mathrm{ALG}_{\mathrm{AL}}$ after chronic stress may contribute to a prolonged negative affective state (28). Importantly, in BNST, STEP is coexpressed in ovBNST CRH neurons and selectively buffers CRH neurons against overactivation after stress (28). While CVMS resulted in increased expression of $\mathrm{CRH}, \mathrm{CRHR} 1$, and PACAP, STEP expression in $\mathrm{BNST}_{\mathrm{ALG}}$ was decreased. These concomitant changes suggest that CVMS disrupts the delicate balance of $\mathrm{CRH}$ signaling and results in hyperactivation of $\mathrm{CRH}$ neurons in the $\mathrm{BNST}_{\mathrm{ALG}}$. Together, our data show that CVMS causes disturbances in BNST CRH signaling that reflect a novel stress-associated molecular signature.

\section{Working Model}

We propose that activation of CRHR1 in ovBNST by CRH stimulates Gs protein-coupled adenylyl cyclase (AC) activity (99), which results in CAMP generation and activation of CAMP-dependent PKA. PKA phosphorylates the KCNQ channel and the GluR1 subunit of AMPA receptors, which mediate M-current inhibition (15) and 
increased mEPSC amplitude $(100,101)$, respectively (Fig.8). CRH itself can also facilitate glutamatergic transmission in many brain regions (102-104). Increased mEPSC amplitude coupled with decreased M-currents contribute to hyperexcitation of ovBNST neurons following chronic stress. In turn, hyperactive ovBNST neurons disrupt neural circuitry and result in dysfunctional HPA axis activation through projections to PVN in the hypothalamus.

Acute optogenetic activation mimicked the effects of CVMS on ovBNST neurons. In these experiments, we utilized dopamine receptor D1a (Drd1a)-Cre transgenic mice where, within BNST, Cre is selectively expressed in ovBNST neurons $(19,37,105)$. Similar to a previous report (Kim et al 2013), ChR2-mediated activation of ovBNST neurons induced negative valence behaviors associated with anxiety. Such acute effects on behavior are likely due to fast stimulatory release of local excitatory neurotransmitters, such as $\mathrm{CRH}$, which could act on the local BNST microcircuitry and through projections to downstream regions such as PVN (6).

\section{Conclusion}

Maladaptive changes of BNST function underlie pathological anxiety disorders in humans (3). Here we identified 1) a novel electrophysiological mechanism suggesting CVMS activates ovBNST and 2) dysfunctional BNST CRH signaling represents a novel chronic stress-associated molecular signature. Activation of ovBNST coupled with neurochemical alterations in $\mathrm{BNST}_{\mathrm{ALG}}$ may result in disturbances that underlie stress-related mood disorders. Electrophysiological and behavioral effects of CVMS were reversed by ovBNST PKA inhibition. Furthermore, optogenetic activation of ovBNST neurons mimicked the effects of CVMS by increasing negative valence behaviors associated with anxiety. Taken together, these data suggest that ovBNST is a critical component of the neural circuitry that underlies stress-induced mood disorders. 


\section{Acknowledgements:}

The authors would like to acknowledge Prof. Tracey J. Shors; Dr. Mimi Phan; Christine Yohn; Frederric Kelada; Kaci Shu; Bren Wu; Ashley Huang; Nicole Jallali; Andrew Dieterich; Ali Yasrebi; and Hannah Wang for helpful discussions and/or technical assistance.

\section{Funding:}

This work was funded by NIMH Grant R01 MH112861 (BAS), NIEHS Grant R21 ES027119 (TAR), NIAAA Grant R01 AA023797 (ZP), and NWO and Alzheimer Nederland (PJL).

\section{Conflict of Interest:}

The authors have no conflicts of interest to declare. 


\section{References:}

1. Choi DC, Furay AR, Evanson NK, Ostrander MM, Ulrich-Lai YM, Herman JP (2007): Bed nucleus of the stria terminalis subregions differentially regulate hypothalamic-pituitary-adrenal axis activity: implications for the integration of limbic inputs. J Neurosci. 27:2025-2034.

2. Davis M, Walker DL, Miles L, Grillon C (2010): Phasic vs sustained fear in rats and humans: role of the extended amygdala in fear vs anxiety. Neuropsychopharmacology. 35:105-135.

3. Ch'ng S, Fu J, Brown RM, McDougall SJ, Lawrence AJ (2018): The intersection of stress and reward: BNST modulation of aversive and appetitive states. Prog Neuropsychopharmacol Biol Psychiatry. 87:108-125.

4. Lebow MA, Chen A (2016): Overshadowed by the amygdala: the bed nucleus of the stria terminalis emerges as key to psychiatric disorders. Mol Psychiatry. 21:450-463.

5. Kash TL, Pleil KE, Marcinkiewcz CA, Lowery-Gionta EG, Crowley N, Mazzone C, et al. (2015): Neuropeptide regulation of signaling and behavior in the BNST. Mol Cells. 38:1-13.

6. Herman JP, Cullinan WE (1997): Neurocircuitry of stress: central control of the hypothalamo-pituitary-adrenocortical axis. Trends Neurosci. 20:78-84.

7. Casada JH, Dafny N (1991): Restraint and stimulation of bed nucleus of the stria terminalis produce similar stress-like behaviors. Brain Res Bull. 27:207-212.

8. Walker DL, Toufexis DJ, Davis M (2003): Role of the bed nucleus of the stria terminalis versus the amygdala in fear, stress, and anxiety. Eur J Pharmacol. 463:199-216.

9. Hammack SE, Richey KJ, Watkins LR, Maier SF (2004): Chemical lesion of the bed nucleus of the stria terminalis blocks the behavioral consequences of uncontrollable stress. Behav Neurosci. 118:443-448.

10. Kageyama K, Suda T (2009): Regulatory mechanisms underlying corticotropin-releasing factor gene expression in the hypothalamus. Endocr J. 56:335-344.

11. Vale W, Spiess J, Rivier C, Rivier J (1981): Characterization of a 41-residue ovine hypothalamic peptide that stimulates secretion of corticotropin and beta-endorphin. Science. 213:1394-1397.

12. Rivier C, Vale W (1983): Modulation of stress-induced ACTH release by corticotropin-releasing factor, catecholamines and vasopressin. Nature. 305:325-327.

13. Bale TL, Vale WW (2004): CRF and CRF receptors: role in stress responsivity and other behaviors. Annu Rev Pharmacol Toxicol. 44:525-557.

14. Morin SM, Ling N, Liu XJ, Kahl SD, Gehlert DR (1999): Differential distribution of urocortin- and corticotropin-releasing factor-like immunoreactivities in the rat brain. 
Neuroscience. 92:281-291.

15. Marrion NV (1997): Control of M-current. Annu Rev Physiol. 59:483-504.

16. Pomrenze MB, Millan EZ, Hopf FW, Keiflin R, Maiya R, Blasio A, et al. (2015): A Transgenic Rat for Investigating the Anatomy and Function of Corticotrophin Releasing Factor Circuits. Front Neurosci. 9:487.

17. Sanderson JL, Dell'Acqua ML (2011): AKAP signaling complexes in regulation of excitatory synaptic plasticity. Neuroscientist. 17:321-336.

18. Sanford CA, Soden ME, Baird MA, Miller SM, Schulkin J, Palmiter RD, et al. (2017): A Central Amygdala CRF Circuit Facilitates Learning about Weak Threats. Neuron. 93:164-178.

19. Daniel SE, Rainnie DG (2016): Stress Modulation of Opposing Circuits in the Bed Nucleus of the Stria Terminalis. Neuropsychopharmacology. 41:103-125.

20. Kim SJ, Park SH, Choi SH, Moon BH, Lee KJ, Kang SW, et al. (2006): Effects of repeated tianeptine treatment on CRF mRNA expression in non-stressed and chronic mild stress-exposed rats. Neuropharmacology. 50:824-833.

21. Dunn JD (1987): Plasma corticosterone responses to electrical stimulation of the bed nucleus of the stria terminalis. Brain Res. 407:327-331.

22. Pomrenze MB, Tovar-Diaz J, Blasio A, Maiya R, Giovanetti SM, Lei K, et al. (2018): A Corticotropin Releasing Factor Network in the Extended Amygdala for Anxiety. $J$ Neurosci.

23. Gungor NZ, Pare D (2016): Functional Heterogeneity in the Bed Nucleus of the Stria Terminalis. J Neurosci. 36:8038-8049.

24. Esteban JA, Shi SH, Wilson C, Nuriya M, Huganir RL, Malinow R (2003): PKA phosphorylation of AMPA receptor subunits controls synaptic trafficking underlying plasticity. Nat Neurosci. 6:136-143.

25. Lee Y, Davis M (1997): Role of the hippocampus, the bed nucleus of the stria terminalis, and the amygdala in the excitatory effect of corticotropin-releasing hormone on the acoustic startle reflex. J Neurosci. 17:6434-6446.

26. Sahuque LL, Kullberg EF, McGeehan AJ, Kinder JR, Hicks MP, Blanton MG, et al. (2006): Anxiogenic and aversive effects of corticotropin-releasing factor (CRF) in the bed nucleus of the stria terminalis in the rat: role of CRF receptor subtypes. Psychopharmacology (Berl). 186:122-132.

27. Dabrowska J, Hazra R, Guo JD, Li C, Dewitt S, Xu J, et al. (2013): Striatal-enriched protein tyrosine phosphatase-STEPs toward understanding chronic stress-induced activation of corticotrophin releasing factor neurons in the rat bed nucleus of the stria terminalis. Biol Psychiatry. 74:817-826.

28. Dabrowska J, Hazra R, Guo JD, Dewitt S, Rainnie DG (2013): Central CRF neurons are not created equal: phenotypic differences in CRF-containing neurons of the rat paraventricular hypothalamus and the bed nucleus of the stria terminalis. Front Neurosci. 7:156.

29. Regev L, Neufeld-Cohen A, Tsoory M, Kuperman Y, Getselter D, Gil S, et al. (2011): 
Prolonged and site-specific over-expression of corticotropin-releasing factor reveals differential roles for extended amygdala nuclei in emotional regulation. Mol Psychiatry. 16:714-728.

30. Sink KS, Walker DL, Freeman SM, Flandreau EI, Ressler KJ, Davis M (2013): Effects of continuously enhanced corticotropin releasing factor expression within the bed nucleus of the stria terminalis on conditioned and unconditioned anxiety. Mol Psychiatry. 18:308-319.

31. Walker DL, Miles LA, Davis M (2009): Selective participation of the bed nucleus of the stria terminalis and CRF in sustained anxiety-like versus phasic fear-like responses. Prog Neuropsychopharmacol Biol Psychiatry. 33:1291-1308.

32. Crestani CC, Alves FH, Correa FM, Guimaraes FS, Joca SR (2010): Acute reversible inactivation of the bed nucleus of stria terminalis induces antidepressant-like effect in the rat forced swimming test. Behav Brain Funct. 6:30.

33. Dong HW, Swanson LW (2004): Organization of axonal projections from the anterolateral area of the bed nuclei of the stria terminalis. J Comp Neurol. 468:277-298.

34. Ju G, Swanson LW (1989): Studies on the cellular architecture of the bed nuclei of the stria terminalis in the rat: I. Cytoarchitecture. J Comp Neurol. 280:587-602.

35. Ju G, Swanson LW, Simerly RB (1989): Studies on the cellular architecture of the bed nuclei of the stria terminalis in the rat: II. Chemoarchitecture. J Comp Neurol. 280:603-621.

36. Cummings S, Elde R, Ells J, Lindall A (1983): Corticotropin-releasing factor immunoreactivity is widely distributed within the central nervous system of the rat: an immunohistochemical study. J Neurosci. 3:1355-1368.

37. Kim SY, Adhikari A, Lee SY, Marshel JH, Kim CK, Mallory CS, et al. (2013): Diverging neural pathways assemble a behavioural state from separable features in anxiety. Nature. 496:219-223.

38. Willner P (2017): The chronic mild stress (CMS) model of depression: History, evaluation and usage. Neurobiol Stress. 6:78-93.

39. Hill MN, Hellemans KG, Verma P, Gorzalka BB, Weinberg J (2012): Neurobiology of chronic mild stress: parallels to major depression. Neurosci Biobehav Rev. 36:2085-2117.

40. Surget A, Saxe M, Leman S, Ibarguen-Vargas Y, Chalon S, Griebel G, et al. (2008): Drug-dependent requirement of hippocampal neurogenesis in a model of depression and of antidepressant reversal. Biol Psychiatry. 64:293-301.

41. Sterrenburg L, Gaszner B, Boerrigter J, Santbergen L, Bramini M, Elliott E, et al. (2011): Chronic stress induces sex-specific alterations in methylation and expression of corticotropin-releasing factor gene in the rat. PLoS One. 6:e28128.

42. Hu P, Liu J, Yasrebi A, Gotthardt JD, Bello NT, Pang ZP, et al. (2016): Gq Protein-Coupled Membrane-Initiated Estrogen Signaling Rapidly Excites Corticotropin-Releasing Hormone Neurons in the Hypothalamic Paraventricular 
Nucleus in Female Mice. Endocrinology. 157:3604-3620.

43. Wang HS, McKinnon D (1995): Potassium currents in rat prevertebral and paravertebral sympathetic neurones: control of firing properties. J Physiol. 485 ( Pt 2):319-335.

44. Joels M, Baram TZ (2009): The neuro-symphony of stress. Nat Rev Neurosci. 10:459-466.

45. Lucassen PJ, Pruessner J, Sousa N, Almeida OF, Van Dam AM, Rajkowska G, et al. (2014): Neuropathology of stress. Acta Neuropathol. 127:109-135.

46. Lucassen PJ, Toni N, Kempermann G, Frisen J, Gage FH, Swaab DF (2019): Limits to human neurogenesis-really? Mol Psychiatry.

47. de Kloet ER, Joels M, Holsboer F (2005): Stress and the brain: from adaptation to disease. Nat Rev Neurosci. 6:463-475.

48. Pego JM, Morgado P, Pinto LG, Cerqueira JJ, Almeida OF, Sousa N (2008): Dissociation of the morphological correlates of stress-induced anxiety and fear. Eur $J$ Neurosci. 27:1503-1516.

49. Waddell J, Morris RW, Bouton ME (2006): Effects of bed nucleus of the stria terminalis lesions on conditioned anxiety: aversive conditioning with long-duration conditional stimuli and reinstatement of extinguished fear. Behav Neurosci. 120:324-336.

50. Somerville LH, Whalen PJ, Kelley WM (2010): Human bed nucleus of the stria terminalis indexes hypervigilant threat monitoring. Biol Psychiatry. 68:416-424.

51. Straube T, Mentzel HJ, Miltner WH (2007): Waiting for spiders: brain activation during anticipatory anxiety in spider phobics. Neuroimage. 37:1427-1436.

52. Kocho-Schellenberg M, Lezak KR, Harris OM, Roelke E, Gick N, Choi I, et al. (2014): PACAP in the BNST produces anorexia and weight loss in male and female rats. Neuropsychopharmacology. 39:1614-1623.

53. Kalin NH, Shelton SE, Fox AS, Oakes TR, Davidson RJ (2005): Brain regions associated with the expression and contextual regulation of anxiety in primates. Biol Psychiatry. 58:796-804.

54. Walker DL, Davis M (2008): Role of the extended amygdala in short-duration versus sustained fear: a tribute to Dr. Lennart Heimer. Brain Struct Funct. 213:29-42. 55. Dhabhar FS, McEwen BS, Spencer RL (1997): Adaptation to prolonged or repeated stress--comparison between rat strains showing intrinsic differences in reactivity to acute stress. Neuroendocrinology. 65:360-368.

56. Odio M, Brodish A (1989): Age-related adaptation of pituitary-adrenocortical responses to stress. Neuroendocrinology. 49:382-388.

57. Pitman DL, Ottenweller JE, Natelson BH (1988): Plasma corticosterone levels during repeated presentation of two intensities of restraint stress: chronic stress and habituation. Physiol Behav. 43:47-55.

58. Viau V, Sawchenko PE (2002): Hypophysiotropic neurons of the paraventricular nucleus respond in spatially, temporally, and phenotypically differentiated manners 
to acute vs. repeated restraint stress: rapid publication. J Comp Neurol. 445:293-307. 59. Choi DC, Evanson NK, Furay AR, Ulrich-Lai YM, Ostrander MM, Herman JP (2008): The anteroventral bed nucleus of the stria terminalis differentially regulates hypothalamic-pituitary-adrenocortical axis responses to acute and chronic stress. Endocrinology. 149:818-826.

60. Yue C, Yaari Y (2004): KCNQ/M channels control spike afterdepolarization and burst generation in hippocampal neurons. J Neurosci. 24:4614-4624.

61. Hernandez CC, Zaika 0, Tolstykh GP, Shapiro MS (2008): Regulation of neural KCNQ channels: signalling pathways, structural motifs and functional implications. $J$ Physiol. 586:1811-1821.

62. Delmas P, Brown DA (2005): Pathways modulating neural KCNQ/M (Kv7) potassium channels. Nat Rev Neurosci. 6:850-862.

63. Robbins J (2001): KCNQ potassium channels: physiology, pathophysiology, and pharmacology. Pharmacol Ther. 90:1-19.

64. Zhou JJ, Gao Y, Kosten TA, Zhao Z, Li DP (2017): Acute stress diminishes M-current contributing to elevated activity of hypothalamic-pituitary-adrenal axis. Neuropharmacology. 114:67-76.

65. Wang S, Yuan F, Li DP (2017): M channels and stress response. Oncotarget. 8:34026-34027.

66. Dell'Acqua ML, Smith KE, Gorski JA, Horne EA, Gibson ES, Gomez LL (2006): Regulation of neuronal PKA signaling through AKAP targeting dynamics. Eur J Cell Biol. 85:627-633.

67. Man HY, Sekine-Aizawa Y, Huganir RL (2007): Regulation of \{alpha\}-amino-3-hydroxy-5-methyl-4-isoxazolepropionic acid receptor trafficking through PKA phosphorylation of the Glu receptor 1 subunit. Proc Natl Acad Sci U S A. 104:3579-3584.

68. Thomas GM, Huganir RL (2004): MAPK cascade signalling and synaptic plasticity. Nat Rev Neurosci. 5:173-183.

69. Song I, Huganir RL (2002): Regulation of AMPA receptors during synaptic plasticity. Trends Neurosci. 25:578-588.

70. O'Brien RJ, Kamboj S, Ehlers MD, Rosen KR, Fischbach GD, Huganir RL (1998): Activity-dependent modulation of synaptic AMPA receptor accumulation. Neuron. 21:1067-1078.

71. Queenan BN, Lee KJ, Pak DT (2012): Wherefore art thou, homeo(stasis)? Functional diversity in homeostatic synaptic plasticity. Neural Plast. 2012:718203.

72. Zadran S, Qin Q Bi X, Zadran H, Kim Y, Foy MR, et al. (2009): 17-Beta-estradiol increases neuronal excitability through MAP kinase-induced calpain activation. Proc Natl Acad Sci U S A. 106:21936-21941.

73. Gao Y, Zhou JJ, Zhu Y, Wang L, Kosten TA, Zhang X, et al. (2017): Neuroadaptations of presynaptic and postsynaptic GABAB receptor function in the paraventricular nucleus in response to chronic unpredictable stress. Br J Pharmacol. 
174:2929-2940.

74. Wang SS, Yan XB, Hofman MA, Swaab DF, Zhou JN (2010): Increased expression level of corticotropin-releasing hormone in the amygdala and in the hypothalamus in rats exposed to chronic unpredictable mild stress. Neurosci Bull. 26:297-303.

75. Davis M, Walker DL, Lee Y (1997): Amygdala and bed nucleus of the stria terminalis: differential roles in fear and anxiety measured with the acoustic startle reflex. Philos Trans R Soc Lond B Biol Sci. 352:1675-1687.

76. Koob GF, Heinrichs SC (1999): A role for corticotropin releasing factor and urocortin in behavioral responses to stressors. Brain Res. 848:141-152.

77. Butler RK, Oliver EM, Sharko AC, Parilla-Carrero J, Kaigler KF, Fadel JR, et al. (2016): Activation of corticotropin releasing factor-containing neurons in the rat central amygdala and bed nucleus of the stria terminalis following exposure to two different anxiogenic stressors. Behav Brain Res. 304:92-101.

78. Faria MP, Miguel TT, Gomes KS, Nunes-de-Souza RL (2016): Anxiety-like responses induced by nitric oxide within the BNST in mice: Role of CRF1 and NMDA receptors. Horm Behav. 79:74-83.

79. Chappell PB, Smith MA, Kilts CD, Bissette G, Ritchie J, Anderson C, et al. (1986): Alterations in corticotropin-releasing factor-like immunoreactivity in discrete rat brain regions after acute and chronic stress. J Neurosci. 6:2908-2914.

80. Stout SC, Mortas P, Owens MJ, Nemeroff CB, Moreau J (2000): Increased corticotropin-releasing factor concentrations in the bed nucleus of the stria terminalis of anhedonic rats. Eur J Pharmacol. 401:39-46.

81. Hammack SE, Cheung J, Rhodes KM, Schutz KC, Falls WA, Braas KM, et al. (2009): Chronic stress increases pituitary adenylate cyclase-activating peptide (PACAP) and brain-derived neurotrophic factor (BDNF) mRNA expression in the bed nucleus of the stria terminalis (BNST): roles for PACAP in anxiety-like behavior. Psychoneuroendocrinology. 34:833-843.

82. Day HE, Curran EJ, Watson SJ, Jr., Akil H (1999): Distinct neurochemical populations in the rat central nucleus of the amygdala and bed nucleus of the stria terminalis: evidence for their selective activation by interleukin-1beta. J Comp Neurol. 413:113-128.

83. Gafford GM, Guo JD, Flandreau EI, Hazra R, Rainnie DG, Ressler KJ (2012): Cell-type specific deletion of GABA(A)alpha1 in corticotropin-releasing factor-containing neurons enhances anxiety and disrupts fear extinction. Proc Natl Acad Sci U S A. 109:16330-16335.

84. Hauger RL, Risbrough V, Oakley RH, Olivares-Reyes JA, Dautzenberg FM (2009): Role of CRF receptor signaling in stress vulnerability, anxiety, and depression. Ann $N$ Y Acad Sci. 1179:120-143.

85. Tran L, Schulkin J, Greenwood-Van Meerveld B (2014): Importance of CRF receptor-mediated mechanisms of the bed nucleus of the stria terminalis in the processing of anxiety and pain. Neuropsychopharmacology. 39:2633-2645. 
86. Elharrar E, Warhaftig G, Issler O, Sztainberg Y, Dikshtein Y, Zahut R, et al. (2013): Overexpression of corticotropin-releasing factor receptor type 2 in the bed nucleus of stria terminalis improves posttraumatic stress disorder-like symptoms in a model of incubation of fear. Biol Psychiatry. 74:827-836.

87. Henckens M, Printz Y, Shamgar U, Dine J, Lebow M, Drori Y, et al. (2017): CRF receptor type 2 neurons in the posterior bed nucleus of the stria terminalis critically contribute to stress recovery. Mol Psychiatry. 22:1691-1700.

88. Hammack SE, Roman CW, Lezak KR, Kocho-Shellenberg M, Grimmig B, Falls WA, et al. (2010): Roles for pituitary adenylate cyclase-activating peptide (PACAP) expression and signaling in the bed nucleus of the stria terminalis (BNST) in mediating the behavioral consequences of chronic stress. $J$ Mol Neurosci. 42:327-340.

89. Hashimoto H, Shintani N, Tanida M, Hayata A, Hashimoto R, Baba A (2011): PACAP is implicated in the stress axes. Curr Pharm Des. 17:985-989.

90. Stroth N, Holighaus Y, Ait-Ali D, Eiden LE (2011): PACAP: a master regulator of neuroendocrine stress circuits and the cellular stress response. Ann N Y Acad Sci. 1220:49-59.

91. Agarwal A, Halvorson LM, Legradi G (2005): Pituitary adenylate cyclase-activating polypeptide (PACAP) mimics neuroendocrine and behavioral manifestations of stress: Evidence for PKA-mediated expression of the corticotropin-releasing hormone (CRH) gene. Brain Res Mol Brain Res. 138:45-57.

92. Hashimoto R, Hashimoto H, Shintani N, Ohi K, Hori H, Saitoh O, et al. (2010): Possible association between the pituitary adenylate cyclase-activating polypeptide (PACAP) gene and major depressive disorder. Neurosci Lett. 468:300-302.

93. Ressler KJ, Mercer KB, Bradley B, Jovanovic T, Mahan A, Kerley K, et al. (2011): Post-traumatic stress disorder is associated with PACAP and the PAC1 receptor. Nature. 470:492-497.

94. Roman CW, Lezak KR, Hartsock MJ, Falls WA, Braas KM, Howard AB, et al. (2014): PAC1 receptor antagonism in the bed nucleus of the stria terminalis (BNST) attenuates the endocrine and behavioral consequences of chronic stress. Psychoneuroendocrinology. 47:151-165.

95. Lezak KR, Roelke E, Harris OM, Choi I, Edwards S, Gick N, et al. (2014): Pituitary adenylate cyclase-activating polypeptide (PACAP) in the bed nucleus of the stria terminalis (BNST) increases corticosterone in male and female rats. Psychoneuroendocrinology. 45:11-20.

96. Kamceva M, Benedict J, Nairn AC, Lombroso PJ (2016): Role of Striatal-Enriched Tyrosine Phosphatase in Neuronal Function. Neural Plast. 2016:8136925.

97. Karasawa T, Lombroso PJ (2014): Disruption of striatal-enriched protein tyrosine phosphatase (STEP) function in neuropsychiatric disorders. Neurosci Res. 89:1-9.

98. Yang CH, Huang CC, Hsu KS (2012): A critical role for protein tyrosine 
phosphatase nonreceptor type 5 in determining individual susceptibility to develop stress-related cognitive and morphological changes. J Neurosci. 32:7550-7562.

99. Grammatopoulos DK (2012): Insights into mechanisms of corticotropin-releasing hormone receptor signal transduction. $\mathrm{Br} J$ Pharmacol. 166:85-97.

100. Malinow R, Malenka RC (2002): AMPA receptor trafficking and synaptic plasticity. Annu Rev Neurosci. 25:103-126.

101. Collingridge GL, Isaac JT, Wang YT (2004): Receptor trafficking and synaptic plasticity. Nat Rev Neurosci. 5:952-962.

102. Liu J, Yu B, Neugebauer V, Grigoriadis DE, Rivier J, Vale WW, et al. (2004): Corticotropin-releasing factor and Urocortin I modulate excitatory glutamatergic synaptic transmission. J Neurosci. 24:4020-4029.

103. Rainnie DG, Bergeron R, Sajdyk TJ, Patil M, Gehlert DR, Shekhar A (2004): Corticotrophin releasing factor-induced synaptic plasticity in the amygdala translates stress into emotional disorders. J Neurosci. 24:3471-3479.

104. Hahn J, Hopf FW, Bonci A (2009): Chronic cocaine enhances corticotropin-releasing factor-dependent potentiation of excitatory transmission in ventral tegmental area dopamine neurons. J Neurosci. 29:6535-6544.

105. Silberman Y, Matthews RT, Winder DG (2013): A corticotropin releasing factor pathway for ethanol regulation of the ventral tegmental area in the bed nucleus of the stria terminalis. J Neurosci. 33:950-960. 
bioRxiv preprint doi: https://doi.org/10.1101/572966; this version posted March 9, 2019. The copyright holder for this preprint (which was not certified by peer review) is the author/funder. All rights reserved. No reuse allowed without permission. 


\section{Figure Legends}

Fig.1: Schematic for experimental design and results for a series of negative valence behavior tests after mice were subjected to chronic variable mild stress (CVMS) paradigm.

(A): Mice were subjected to a 6-week chronic variable mild stress (CVMS) paradigm, then subjected to a series of anxiety/depressive-like behavioral tests including sucrose preference test, elevated plus maze (EPM) test, open field (OF) test, novelty suppression feeding (NSF) test and forced swimming test, then were perfused for immunohistochemistry (IHC) study.

(B): Duration in open arm compared between control ( $n=10)$ vs. CVMS $(n=10)$ mice in the elevated plus maze (EPM) test.

(C): Frequency in the open arm compared between control $(n=10)$ vs. CVMS $(n=10)$ mice in the elevated plus maze (EPM) test.

(D): Sucrose preference percentage compared between control $(n=10)$ vs. CVMS $(\mathrm{n}=10)$ mice.

(E): The distance that the mice travelled in the center of open field (OF) test compared between control $(n=10)$ vs. CVMS $(n=10)$ mice.

(F): The duration that the mice spent in the center of open field (OF) test compared between control $(n=10)$ vs. CVMS $(n=10)$ mice.

$(G)$ : The frequency that the mice entried into the center of open field (OF) test compared between control $(n=10)$ vs. CVMS $(n=10)$ mice.

$(\mathrm{H})$ : Comparison of the latency to eat food pellets in the novelty suppressed feeding (NSF) test between control $(n=10)$ vs. CVMS $(n=10)$ mice.

(I): Comparison of the immobility time in the forced swim test (FST) between control $(n=10)$ vs. CVMS ( $n=9)$ mice.

$\mathrm{n}=9-10$ animals per group; ${ }^{* *}: \mathrm{p}<0.01 ;^{* * *}: \mathrm{p}<0.001$.

Fig.2: Anatomy example of mice oval nucleus of the bed nucleus of the stria terminalis (ovBNST) and chronic variable mild stress (CVMS) effects on the electrophysiological properties (including $\mathrm{M}$-current recording and miniature excitatory postsynaptic current (mEPSC)) in the mice ovBNST.

(A): Anatomy example of ephys recording site in the oval nucleus of mice bed nucleus of the stria terminalis (ovBNST) by whole-cell patch clamping method; ic: internal capsule; ac: anterior commissure.

(B): Left: I-V plot of M-current from $-75 \mathrm{mV}$ to $-25 \mathrm{mV}$ shows that M-current recorded in ovBNST did not run down after $20 \mathrm{~min}$; Right: example of M-current traces shown at $0 \mathrm{~min}, 10 \mathrm{~min}$ and $20 \mathrm{~min}$ and after subsequent perfusion with KCNQ-selective channel blocker XE991 (10 min, $40 \mu \mathrm{M})$.

(C): The deactivation protocol used to record the M-current: from a holding potential of $-60 \mathrm{mV}$, a voltage jump to $-20 \mathrm{mV}$ (300 ms) was followed by steps from 
$-30 \mathrm{mV}$ to $-75 \mathrm{mV}$ in $5 \mathrm{mV}$ increments (500 ms);

(D): An example of continuous action potential firing activity in a recorded ovBNST neuron during perfusion of selective KCNQ channel blocker XE991 (40 $\mu \mathrm{M})$ for 10 min after 2 min baseline recording under current-clamp mode. Robust action potential is seen starting $7 \mathrm{~min}$ after XE991 perfusion.

(E): I-V plot shows significantly diminished outward M-current in ovBNST neurons ranging from $-75 \mathrm{mV}$ to $-25 \mathrm{mV}$ compared between control vs. CVMS mice (both $n=6$ cells). Significant difference was found between the voltage range of -50 to -25 $\mathrm{mV}$.

(F): Comparison of cellular resting membrane potential (RMP) in ovBNST neurons from control ( $\mathrm{n}=6$ cells) vs. CVMS mice ( $\mathrm{n}=5$ cells) revealed significant higher RMP in the ovBNST of CVMS mice.

(G): Comparison of cellular input resistance (IR) in ovBNST neurons from control ( $n=5$ cells) vs. CVMS mice ( $n=5$ cells) revealed significant higher IR in the ovBNST of CVMS mice.

(H): Example of a comparison of mEPSC traces in ovBNST from control vs. CVMS mice.

(I): Average mEPSC amplitude increased in ovBNST of CVMS mice ( $\mathrm{n}=6$ cells) compared to Control mice ( $\mathrm{n}=5$ cells).

(J): Average mEPSC frequency did not change in ovBNST of CVMS mice ( $\mathrm{n}=6$ cells) compared with Control mice ( $\mathrm{n}=5$ cells).

*: $p<0.05$; $^{* *}: \mathrm{p}<0.01$; NS: non-significant different $(\mathrm{p}>0.05)$.

Fig.3.1: Typical example of high magnification figure showing immunostaining of CRH (A), c-fos (B), PACAP (C) and STEP (D) in the mice oval nucleus of BNST (ovBNST).

(A): White arrows point to typical CRH-immunoreactive (IR) cells in the ovBNST:

(B): White arrows point to typical c-fos-immunoreactive (IR) cells in the ovBNST;

(C): White arrows point to typical PACAP-immunoreactive (IR) cells in the ovBNST:

(D): White arrows point to typical STEP-immunoreactive (IR) cells in the ovBNST:

All figures are in $40 \mathrm{X}$ magnification. Scale bar: $50 \mu \mathrm{m}$. ic: internal capsule; ac: anterior commissure.

Fig.3.2: Comparison of c-fos (A,B), CRH (C,D), PACAP (E,F) and STEP (G,H)immunoreactive (IR) cell numbers in both whole antero-dorsolateral BNST (BNSTadl) and the oval nuclei of BNST (ovBNST).

(A) and (B): Comparison of c-fos-IR cell number in the (A) BNST adl and (B) ovBNST shows higher number of c-fos-IR cells in both regions of BNST from CVMS mice $(n=6)$ compared to Control mice $(n=6) ; .$.

(C) and (D): Comparison of CRH-IR cell number in the (C) BNST adl and (D) ovBNST shows higher number of CRH-IR cells in both regions of BNST from CVMS mice $(n=6)$ 
compared to Control mice $(\mathrm{n}=6)$;

(E) and (F): Similarly, comparison of PACAP-IR cell number in the (E) BNST adl and (F) ovBNST shows higher number of PACAP-IR cells in both regions of BNST from CVMS mice $(n=6)$ compared to Control mice $(n=6)$;

$(G)$ and $(H)$ : On the contrary, comparison of STEP-IR cell number in the (G) BNST adl and $(H)$ ovBNST reveals lower number of STEP-IR cells in both regions of BNST from CVMS mice $(n=6)$ compared to Control mice $(n=6)$.

${ }^{* *}: \mathrm{p}<0.01 ;{ }^{* * *}: \mathrm{p}<0.001$

Fig.4: Schematic experimental design and results for qPCR and western blot study after mice were subjected to 6 -week chronic variable mild stress (CVMS) paradigm.

(A): Experimental design for qPCR and western blot study to detect mRNA and protein expression changes in the antero-dorsal lateral region of the BNST (BNST adl $_{\text {) }}$ after 6-week CVMS paradigm.

(B): Higher expression of CRH mRNA was found in BNSTadl of CVMS mice (n=7) vs. control mice $(\mathrm{n}=9)$;

(C): Similarly, PACAP mRNA expression was higher in BNSTadl from CVMS $(n=7)$ mice vs. control ( $\mathrm{n}=7)$ mice;

(D): On the contrary, comparison of STEP mRNA expression in BNSTadl between control ( $n=9$ ) vs. CVMS ( $n=9$ ) mice revealed lower STEP mRNA expression from CVMS mice;

(E): CRHR1 mRNA expression in BNSTadl of CVMS $(n=8)$ mice is higher compared to control ( $\mathrm{n}=9$ ) mice;

(F): No significant change was found for the mRNA expression of CRHR2 in BNSTadl compared between control $(n=7)$ vs. CVMS $(n=9)$ mice;

(G): Example of western blot example showing comparison of $42 \mathrm{kDa}$ phospho-PKA C (Thr197) ( $1^{\text {st }}$ lane) and $42 \mathrm{kDa}$ PKA (C- $\left.\alpha\right)\left(2^{\text {nd }}\right.$ lane) in BNSTadl from control (Left; $\mathrm{n}=6$ ) vs. CVMS (Right; $\mathrm{n}=6$ ) mice; $37 \mathrm{kDa}$ GAPDH was used as endogenous control;

(H): Example of western blot example showing comparison of expression of $61 \mathrm{kDa}$ membrane isoform of STEP61 and $46 \mathrm{kDa}$ cytosolic isoform of STEP46 in BNSTadl from control $(n=6)$ vs. CVMS $(n=6)$ mice; $37 \mathrm{kDa}$ GAPDH was used as endogenous control;

(I): Quantification of PKA protein expression level in BNSTadl between control $(n=6)$ vs. CVMS ( $\mathrm{n}=6)$ mice;

(J): Quantification and comparison of p-PKA (Thr197) expression level in BNSTadl from control $(n=6)$ vs. CVMS $(n=6)$ mice revealed higher expression of $p$-PKA from the CVMS mice compared to Control mice;

(K): Quantification of comparison of cytosolic STEP46 revealed higher expression level in the BNSTadl from control $(n=6)$ vs. CVMS $(n=6)$ mice;

(L): Quantification of comparison of membrane STEP61 expression level in BNSTadl 
from control ( $n=6)$ vs. CVMS $(n=6)$ mice revealed no significance change compared between these 2 groups.

*: $\mathrm{p}<0.05 ;{ }^{* * *}: \mathrm{p}<0.001$;

NS: non-significant different $(p>0.05)$.

Fig.5: Effects of CVMS on the M-currents and mEPSC recorded in the ovBNST are partly mediated by PKA-activation.

(A): BNST brain slices from CVMS mice were pre-incubated with PKA-selective antagonist $\mathrm{H} 89$ for 30 min to compare its effect on the properties of M-current and mEPSC recorded in the oval nucleus of BNST (ovBNST) of the chronic stress (CVMS) mice. I-V curve of outward M-current shows that the attenuated M-currents in ovBNST of CVMS mice were partly normalized at the membrane voltage ranging from $-45 \mathrm{mV}$ to $-25 \mathrm{mV}$ after slices were pre-incubated with PKA-selective antagonist $\mathrm{H} 89$ ( $\mathrm{n}=6$ cells).

(B): H89 ( $n=6$ cells) pre-incubation normalized the increased mEPSC amplitude in ovBNST of CVMS mice ( $\mathrm{n}=5$ cells) compared with Control mice.

(C): H89 ( $n=6$ cells) pre-incubation had no significant effect on the mEPSC frequency in ovBNST of CVMS mice ( $\mathrm{n}=5$ cells).

*: $\mathrm{p}<0.05 ;{ }^{* * *}: \mathrm{p}<0.001$;

NS: non-significant different $(p>0.05)$.

Fig.6: PKA activation in ovBNST mediates CVMS effects on negative valence behaviors associated with anxiety.

(A): Cannula surgery schematic showing that PKA-selective antagonist H89 was chronically infused into the oval nucleus of BNST (ovBNST) of CVMS mice for 7 days continually to assess behavioral effects of CVMS in EPM, OF, SPT, and NSF test). CVMS exposure was continuously present during the chronic 7-day drug infusion period. Mice was allowed to recover for 7 days before chronic H89 injection starts after cannula surgery.

(B): Anatomy example shows cannula inserted into ovBNST.

(C): PKA-selective antagonist H89 (Stress+H89; $n=7$ ) significantly increased the duration time that mice spent in open arm of EPM test compared with CVMS mice (Stress; $n=10$ ).

(D): H89 (Stress+H89; $n=7$ ) had no significant effect on the frequency that mice entried into open arm of EPM test compared with CVMS mice (Stress; $n=10$ ).

(E): H89 (Stress+H89; $n=7$ ) significantly increased the distance that the CVMS mice (Stress; $n=10$ ) traveled in the center of open field (OF) test.

(F): H89 (Stress+H89; n=7) significantly increased the duration that the CVMS mice (Stress; $n=10$ ) spent in the center of open field (OF) test.

(G): H89 (Stress+H89; $n=7$ ) significantly increased the frequency that the CVMS 
mice (Stress; $n=10$ ) entried in the center of open field (OF) test.

(H): H89 (Stress+H89; $n=7$ ) significantly increased the sucrose preference percentage of CVMS mice (Stress; $\mathrm{n}=10$ ).

(I): H89 (Stress+H89; n=7) significantly decreased the latency that the CVMS mice (Stress; $n=10$ ) start to eat food pellet of novelty suppressed feeding (NSF) test.

\section{Fig.7: Optogenetically stimulating oval nucleus in the BNST (ovBNST) promotes negative valence behaviors associated with anxiety in EPM and OF.}

(A): Schematic graph showing the timeline of opsin virus (AAV5-EF1 $\alpha$-DIO-ChR2 (H134R)-eYFP, ChR2) or control virus (AAV5-EF1 $\alpha$-DIO-eYFP, Control) injected into ovBNST of dopamine receptor D1a (Drd1a)-Cre transgenic mice. Mice were allowed to undergo at least 3 weeks recovery before subjected to either EPM or OF test (both composed of 3 different sessions of lights off-on-off series) under blue light stimulation.

(B) and (C) shows the expression of Control-eYFP virus (B) and ChR2-eYFP virus (C) in ovBNST. Scale bar: $400 \mu \mathrm{m}$. Ic: internal capsule; ac: anterior commissure; ov: oval nucleus.

(D): Comparison of the duration time that Control $(n=8)$ mice vs. ChR2 ( $n=8)$ mice spent in open arm during the OFF-ON-OFF session (each $5 \mathrm{~min}$ ) in EPM test revealed decreased open arm duration when blue light on only in the ChR2 group but not in the Control group.

(E): Comparison of the frequency that Control $(n=8)$ mice vs. ChR2 $(n=8)$ mice entered into open arm during the OFF-ON-OFF session (each $5 \mathrm{~min}$ ) in EPM test revealed decreased open arm frequency when blue light on only in the ChR2 group but not in the Control group.

(F): Comparison of the distance that Control $(n=7)$ mice vs. ChR2 $(n=8)$ mice traveled in the center area during the OFF-ON-OFF session (each $10 \mathrm{~min}$ ) in open Field test revealed decreased traveling distance in the OF center when blue light on only in the ChR2 group but not in the Control group.

$(G)$ : Comparison of the time duration that Control $(n=7)$ mice vs. ChR2 $(n=8)$ mice spent in the center area during the OFF-ON-OFF session (each $10 \mathrm{~min}$ ) in open Field test revealed decreased duration spent in the OF center when blue light on only in the ChR2 group but not in the Control group.

(H): Comparison of the frequency that Control $(n=7)$ mice vs. ChR2 $(n=8)$ mice entried into the center area during the OFF-ON-OFF session (each $10 \mathrm{~min}$ ) in open Field test revealed decreased entry frequency into the OF center when blue light on only in the ChR2 group but not in the Control group.

$*: \mathrm{p}<0.05$;

$* *: \mathrm{p}<0.01$.

Fig.8: Working model illustrating the sequence of the molecular cascades of 


\section{the CRH stress signaling in the BNST.}

Chronic stress 1): induces CRH production and release in the BNST, which sequentially activates CRHR1, a Gs-protein coupled membrane receptor. 1) Activation of AC (adenylyl cyclase) (coupled to Gs) then generates 2) cAMP production, which in turn 2): activates PKA enzyme. Activation of PKA then initiates two parallel phosphorylation pathways:

3) phosphorylates KCNQ channel on the cellular membrane to mediate inhibition of the M-current; and

4) phosphorylates GluR1 subunit of AMPAR on the postsynaptic membrane to mediate potentiation of mEPSC amplitude.

These two parallel pathways converge together to mediate M-current inhibition and increased mEPSC amplitude.

Meanwhile: 5) PACAP (function as CRH activator) and STEP (function as CRH inhibitor) neuropeptide will function to activate and inhibit CRH respectively. 


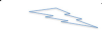

\section{2 week oge}

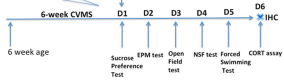

B

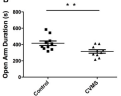

C

ns

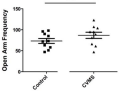

D

E

F

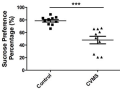

G

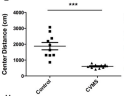

H
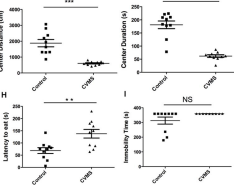


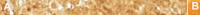

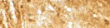

\section{8.

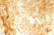

the

if

CRH

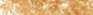

ifot

CRH

\section{c-fos}
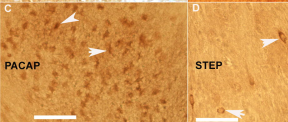

STEP
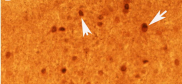

$+-7$

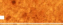

(avs

(5) 10

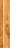

19

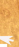

4.
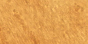


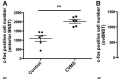

t

.
B $\quad \cdots \quad$ C $\quad$ =

D
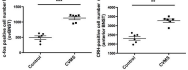

$\frac{1}{2}$

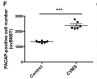

.

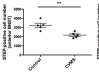

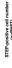

r.

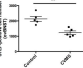




\section{A 6-week cums}

\section{blood CORT test + qPCR + Western blot}

$\mathrm{B}$

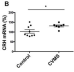

0

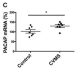

cres

-
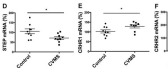

icli
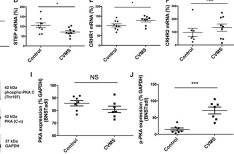

\section{H Dented}

sove

$--$

$-m$

simas

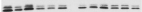

ans. $\sin$

มา ienow

k

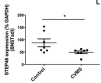

L.

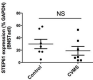


A

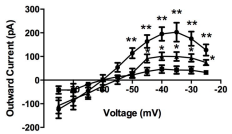

$\rightarrow$ Control $(n=6)$

- CVMS $(n=6)$

- CVMS+H89 $(n=6)$
B

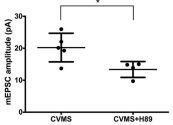

C

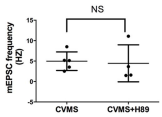




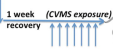

H89 injection

into ovBNST for 7 days

EPM test

Open Field test

Sucrose Preference test

NSF test

B

C

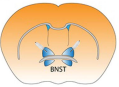

E

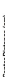

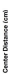
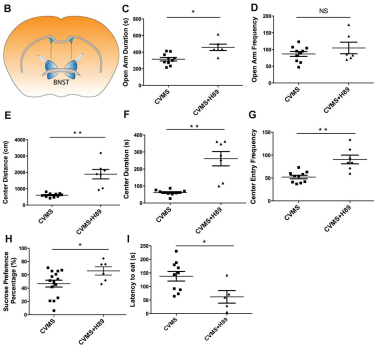

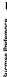
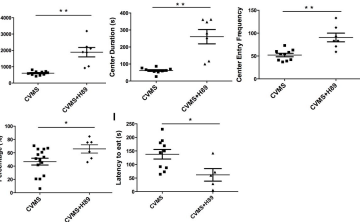


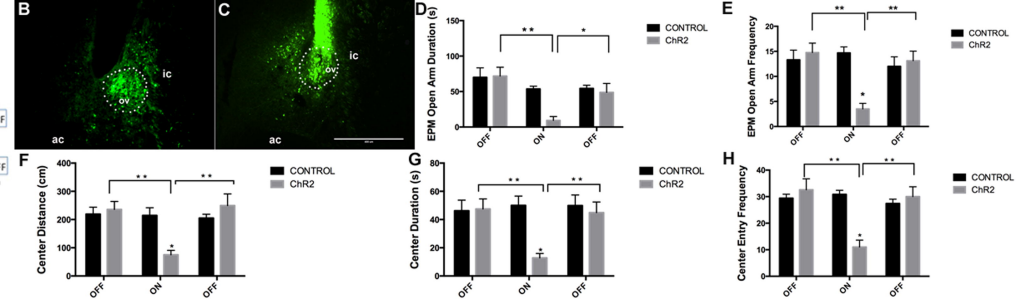




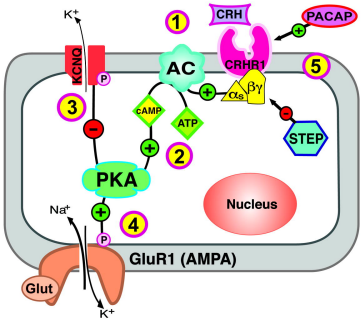

